\title{
A VERTICAL MICROMACHINED RESISTIVE HEATER FOR A MICRO GAS SEPARATION COLUMN
}

\author{
Janet K. Robertson \\ Standard MEMS Inc. \\ Burlington, MA 01803
}

\begin{abstract}
This paper presents a micromachined vertical resistive heater fabricated as part of a micro gas separation column. The complete device is formed from two silicon die electrostatically bonded to a single glass die. The glass die is conventionally machined and contains four through holes. The holes are filled with a zeolite adsorbent and thus act as separation columns. Each silicon die serves as a screen which allows air to flow through the device while holding the zeolite in place. The $5000 \mu \mathrm{m}$ diameter screens are Deep Reactive Ion Etched (DRIE) and contain 1964 square $50 \mu \mathrm{m} \times 50 \mu \mathrm{m} \times 350 \mu \mathrm{m}$ channels. A vertical resistor is also integrated onto one of the silicon die. The junction isolated resistor is formed from a phosphorus diffusion which occurs after DRIE. Thus, the resistor is embedded into the walls of the micromachined silicon screen. The vertical resistor is used to heat a purge gas and regenerate the zeolite adsorbent. The measured vertical resistance is between $6 \Omega$ and $7 \Omega$. The residence time of the gas in the heater is $\sim 60 \mathrm{msec}$ assuming a $1 \mathrm{SCCM} \mathrm{N} 2$ gas flow rate. The theoretical heat transfer coefficient is $3000 \mathrm{~W} / \mathrm{m}^{2} /{ }^{\circ} \mathrm{C}$. The power consumed to heat both the screen and the gas at steady state is $\sim 33 \mathrm{~mW} /$ die.
\end{abstract}

\section{INTRODUCTION}

The development of a micro gas separation system is a key component for the continued development of micro chemical processes. Like micro valves [1], pumps [2] and heat exchangers [3], the development of a micro gas separation system would contribute greatly to the successful development of a microreactor process. For example, a separation system could remove contaminants from feed gas entering a micro reactor and/or remove unwanted by-products from the effluent.

A micro separation system could also be used to enhance the performance of gas sensors. Current gas sensors, while having good sensitivity, suffer from poor selectivity, responding equally well to a number of different gas species. By incorporating one or more gas separation columns in the incoming flow stream, species which are not of interest could be removed, reducing the possibility of ambiguous gas sensor responses. A gas separation/gas sensing system could then be used to control a micro chemical process by the real-time separation and detection of critical product gases.

Although many separations are possible, the prototype micro gas separation column was designed to separate water from air. The column uses $5 \mathrm{~A}$ powdered zeolite to dry 1SCCM of air. The on-stream time (i.e. the time necessary to saturate the adsorbent with water) at $25^{\circ} \mathrm{C}$ and $100 \%$ humidity is approximately 1 hour. The zeolite is regenerated by passing a heated purge gas through the separation column, heating the zeolite and desorbing the water. By using multiple columns, some adsorbing while others are regenerating, the system can supply a continuous stream of dry air. The zeolite is completely regenerated when the bed temperature reaches $350^{\circ} \mathrm{C}$. Therefore, the purge gas needs to be heated to at least $350^{\circ} \mathrm{C}$.

This paper primarily describes one part of the micro gas separation system, the vertical micromachined resistive heater.

\section{DEVICE FABRICATION}

The micro gas separation column, shown in Figure 1, is fabricated from two silicon die electrostatically bonded to a single glass die. The completed device is approximately $1.7 \mathrm{~cm} \times 1.7 \mathrm{~cm}$. The glass die is conventionally machined ${ }^{1}$ and contains four through holes, which form the separation columns and house the zeolite adsorbent. Each silicon die is micromachined using Deep Reactive Ion Etching (DRIE) to produce a silicon screen which allows gas to flow through the device but prevents the powered zeolite from escaping. One of the two silicon die (the silicon heater die) also serves to heat the purge gas. It contains a junction isolated vertical resistive heater formed by a phosphorus diffusion which occurs after the die has been micromachined. The valves necessary for cyclic regeneration of the zeolite are not integrated with the device.

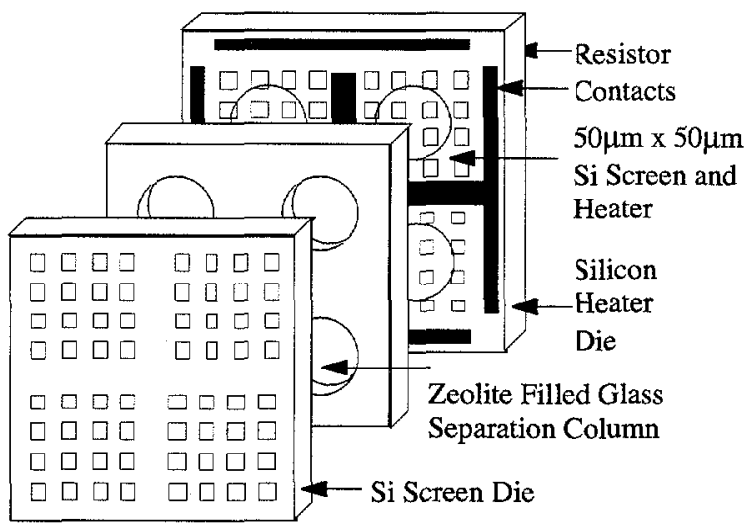

Figure 1. Perspective drawing of the Micro Gas Separation Column.

The resistive heater is diffused into the walls of the silicon micromachined screen. Thus, current flows vertically from the frontside $\mathrm{Al}$ contacts through the resistor to the backside $\mathrm{Al}$ contacts. Therefore, the device is inherently double-sided. The fabrication steps described below, for the resistive heater, are performed on both the back and the front of 3inch double-side polished 100 silicon wafers. The one exception is the DRIE which proceeds from one side and stops on PECVD layers deposited only on the opposite side. To facilitate connection to

\footnotetext{
I The glass wafers and the glass shadow masks were manufactured at Specialty Glass Products, Willow Grove, PA.
} 
both the frontside and the backside contacts, the silicon heater die is larger than either the glass die or the silicon screen die.

Fabrication of the resistive heater, shown in Figure 2, begins with a photostep which patterns photoresist for a $5000 \AA$ dry plasma recess. The recess facilitates electrostatic bonding of the silicon die to the glass separation columns by recessing areas of the silicon containing steps formed from subsequent selective diffusions. The recess was omitted from the fabrication of the prototype resistive heater since these die were intended to test the function of the heater alone.

Following recess, a $1,2 \mu \mathrm{m}$ wet thermal masking oxide is grown and patterned for $\mathrm{n}+$ contact diffusion. Following diffusion, the masking oxide is stripped and a new thermal oxide grown and patterned for Boron Ion Implant. After the oxide has been etched, a second thin dry oxide is grown. This oxide moves the peak of the implant doping distribution toward the surface of the silicon facilitating the formation of an ohmic contact to the lightly doped p- substrate. After Implant all oxide is removed with BHF.

The silicon screen is formed by dry etching $50 \mu \mathrm{m} \times 50 \mu \mathrm{m}$ square channels completely through the silicon wafer. After this etch is complete, photoresist processing is far more difficult because of the presence of these through holes. Therefore, the masking oxide that will be needed after DRIE is grown and patterned before DRIE. Thus, following the removal of the implant masking oxide, $6000 \AA$ of wet thermal oxide is grown and patterned. This 'block' oxide will be used to mask the phosphorus resistor diffusion.

After the block oxide has been patterned, $5000 \AA$ of PECVD silicon nitride and $1 \mu \mathrm{m}$ of PECVD silicon dioxide are deposited on one side of the wafer. On the opposite side, a $9 \mu \mathrm{m}-10 \mu \mathrm{m}$ thick layer of positive photoresist is patterned for the $50 \mu \mathrm{m} \times 50 \mu \mathrm{m}$ through holes. Following plasma descum, the wafer is etched for $\sim 4$ hours in a Bosch etcher ${ }^{2}$. The etch proceeds through the silicon wafer, through the PECVD nitride and stops on the PECVD oxide. The stopping oxide is necessary to prevent the plasma from attacking the exposed silicon surrounding the through hole on the underneath side of the wafer. This type of attack, or etching, is sometimes called "spidering" because the etch pattern spiders out in all directions around the exit hole. A perspective of the micromachined through holes is shown in Figure 3. SEM photographs of the silicon screen are shown in Figures 4-6.

The PECVD oxide and nitride layers are then removed. This can be accomplished using either wet or dry etching processes. If dry etching is used, the frontside of the wafer resists etching since it is face down in the plasma and still covered with photoresist. Alternately, BHF can be used to remove the PECVD oxide. The photoresist is then stripped and hot phosphoric used to remove the PECVD nitride. Regardless, the block oxide on both sides of the wafer is unaffected.

It is important that the resistance of the $n+$ vertical resistor be larger than the resistance of the $\mathrm{Al}$ interconnects. The majority of the applied voltage should drop across the vertical resistor generating heat primarily in the silicon screen. Therefore, following DRIE, $600 \AA$ of dry oxide is grown on the wafer. The oxide will grow not only on the surface of the wafer but also on the surface of the square through holes. The POCOL diffusion then takes place through the oxide which lowers the

\footnotetext{
${ }^{2}$ The Bosch etching was performed at the NNUN facility at Cornell University.
}

dopant concentration. Note that obtaining an exact dopant concentration is not critical. It is only important that the impedance of the embedded resistor be larger than the interconnect impedance.
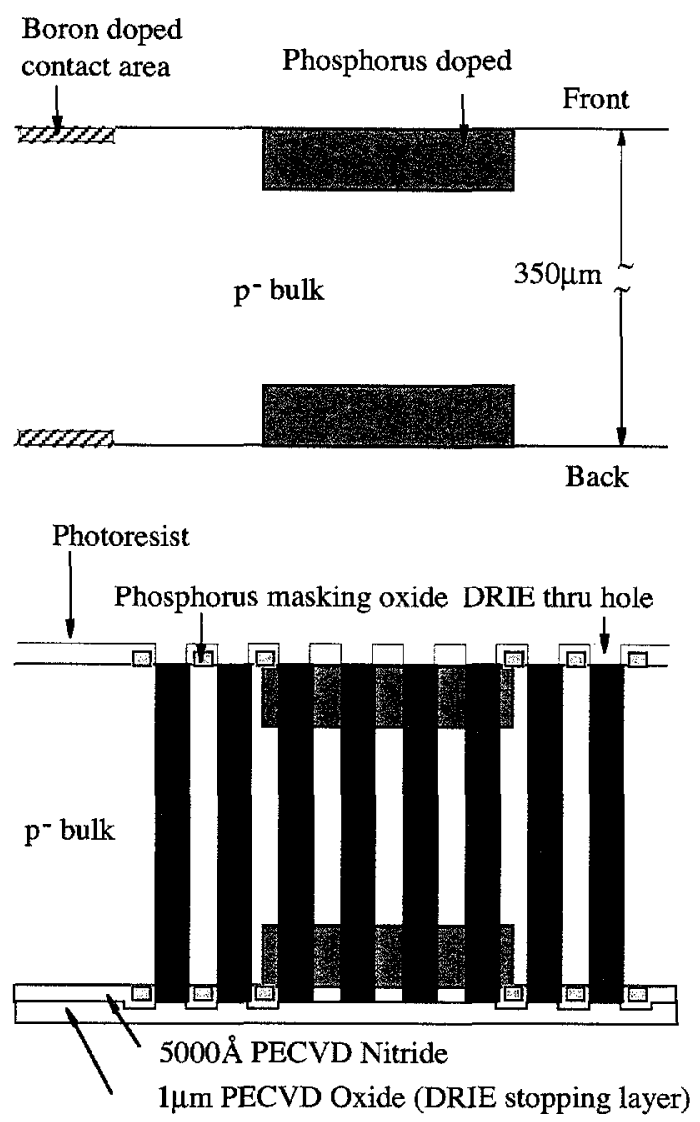

Silicon is removed in these areas to reduce the thermal mass of the surrounding structure

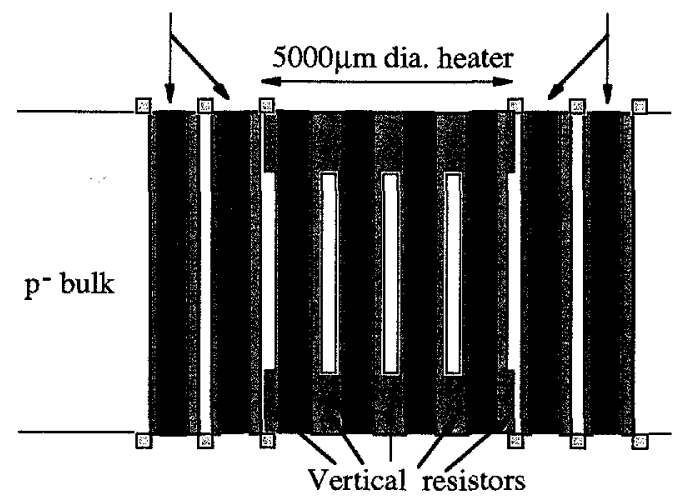

Figure 2. Fabrication Sequence for the Silicon Heater Die.

The silicon screen extends beyond the boundary of the circular glass separation column. This helps to lower the total thermal mass of the silicon die. However, it is undesirable for additional heat to be generated in this area. The block oxide is used to electrically isolate the corners of the silicon screen from the embedded resistor. Without this oxide, the entire surface of 
the wafer would be doped $n+$ during the resistor POCOL diffusion. The through holes in the corners of the silicon screen would then become part of the embedded resistor. The block oxide is patterned in a square picture frame $(15 \mu \mathrm{m}$ wide) around each DRIE through hole in the corners of the silicon screen. This prevents the resistor phosphorus diffusion from doping these areas, electrically isolating them from the embedded resistor.

After completion of the resistor phosphorus diffusion, the block oxide is removed in BHF. A glass shadow mask is then aligned and clamped to the silicon wafer. The alignment tolerances and the metal contacts are large which facilitates accurate alignment by hand through a microscope. The clamping fixture was designed to be easily inserted into a metal evaporator. $1 \mu \mathrm{m}$ of $\mathrm{Al}$ is then deposited. The procedure is repeated to form the hackside contacts.

Fabrication of the silicon screen die begins with the growth of $\sim 1 \mu \mathrm{m}$ of wet thermal oxide. The oxide is then selectively removed from the front side of the silicon wafer using BHF. The front of the wafer is then coated with $9 \mu \mathrm{m}-$ $10 \mu \mathrm{m}$ of positive photo resist, exposed and developed opening $50 \mu \mathrm{m} \times 50 \mu \mathrm{m}$ square holes. Following a plasma descum the wafers were etched in a Bosch etcher for $\sim 4$ hours. Removal of the photoresist and masking layers completes the process.

The glass die are machined from four inch, $800 \mu \mathrm{m}$ thick, borosilicate glass wafers. The columns are $5000 \mu \mathrm{m}$ in diameter and $800 \mu \mathrm{m}$ long. If larger zeolite volumes are desired, to accommodate higher gas flow rates, both the thickness of the glass and diameter of the columns can be increased.

\section{Calculations}

The following analysis was developed to estimate the heat transfer coefficient and the steady state power required to heat the purge gas to approximately $400^{\circ} \mathrm{C}$. At steady state, the vertical resistors generate a nearly constant heat flux along the wall of each vertical channel. The bulk temperature of the gas increases linearly as it flows through the heater. An energy balance written for the control volume shown in Figure 7 yields

$$
q \cdot 4 s \Delta x+m c_{p}\left(T_{\left.B\right|_{x}}-T_{r e f}\right)-m c_{p}\left(T_{\left.B\right|_{x+\Delta x}}-T_{r e f}\right)=0
$$

where $q$ is the heat generated per unit time per unit of surface area, $m$ is the mass flow rate, $c_{p}$ the specific heat at constant pressure, and $s=50 \mu m$ is the side length of the square channel. In the limit as $\Delta x \rightarrow 0$

$$
\frac{d T_{B}}{d x}=\frac{4 s q}{m c_{p}}
$$

Integrating (2) provides the average, or mixing-cup temperature of the purge gas as it travels down the length of the tube;

$$
T_{B}=T_{i}+\frac{4 s q}{m c_{p}} x
$$

where $T_{B}$ is the temperature of the gas in the tube at position $x$ and $T_{i}$ is the temperature of the gas at the inlet. Newton's Law of Cooling states that the local heat flux is proportional to the local temperature difference, i.e.

$$
q=h\left(T_{w}-T_{B}\right)
$$

where $h$ is the heat transfer coefficient and $T_{w}$ is the wall temperature. Clearly both $T_{w}$ and $T_{B}$ vary linearly down the length of the channel. Combining Equations (3) and (4) yields the following expression for the silicon wall temperature as a function of the gas inlet temperature and the position $x$ along the channel

$$
T_{w}=T_{i}+q\left[\frac{1}{h}+\frac{4 s x}{m c_{p}}\right]
$$

For flow with a fully developed velocity and thermal profile, and a constant wall flux, $h$ is given by [4]

$$
h=4.364 \frac{k_{\text {gas }}}{D}
$$

where $D$ is the hydraulic diameter and $k_{\text {gas }}$ is the thermal conductivity of the gas. Therefore, using Tables 1 and 2 , the calculated heat transfer coefficient is $3000 \mathrm{~W} / \mathrm{m}^{2} /{ }^{\circ} \mathrm{C}$.

The total power necessary to continuously heat a $1 \mathrm{SCCM}$ flow of nitrogen from $25^{\circ} \mathrm{C}$ to $400^{\circ} \mathrm{C}$ can be calculated by first calculating the heat flux at steady state. Using Equation (3) $q=67.7 \mathrm{~W} / \mathrm{m}^{2}$. The total interior surface area of a single channel is $6.2 \cdot 10^{-8} \mathrm{~m}^{2}$. Thus, the heat generation rate per channel is $4.2 \mu \mathrm{W} / \mathrm{channel}$. The total power required to heat the purge gas is $8.24 \mathrm{~mW} /$ bed.

The heater will not only heat the gas flowing through the channels but will also conductively heat the silicon die. The Biot number is the ratio of surface convection to internal conduction. For the silicon screen the Biot number is 0.18 , which implies that the screen heats uniformly. The heat required to raise the silicon screen temperature from $25^{\circ} \mathrm{C}$ to $400^{\circ} \mathrm{C}$ is $3.11 \mathrm{~J}$.

Table 1. Geometry of the Silicon Screen Channel side length $\quad 50 \mu \mathrm{m}$ Hydraulic diameter $\quad 56.42 \mu \mathrm{m}$ Channel length $\quad 350 \mu \mathrm{m}$ Number of channels per bed $\quad 1963$ Diameter of the bed $\quad 5000 \mu \mathrm{m}$ Number of beds per die $\quad 4$

Table 2. Physical Properties of N2 Inlet temperature $\quad 25^{\circ} \mathrm{C}$ Outlet temperature $\quad 400^{\circ} \mathrm{C}$ Heat capacity $\quad 1.06 \mathrm{~kJ} / \mathrm{kg} /{ }^{\circ} \mathrm{K}$ Thermal conductivity $\quad 38.7 \mathrm{~mW} / \mathrm{m} /{ }^{\circ} \mathrm{C}$ Mass flow rate $2.08 \cdot 10^{-8} \mathrm{~kg} / \mathrm{sec}$

\section{PRELIMINARY TEST RESULTS}

The measured vertical resistance of the heater die is between $6 \Omega$ and $7 \Omega$ with the p- substrate floating. The 


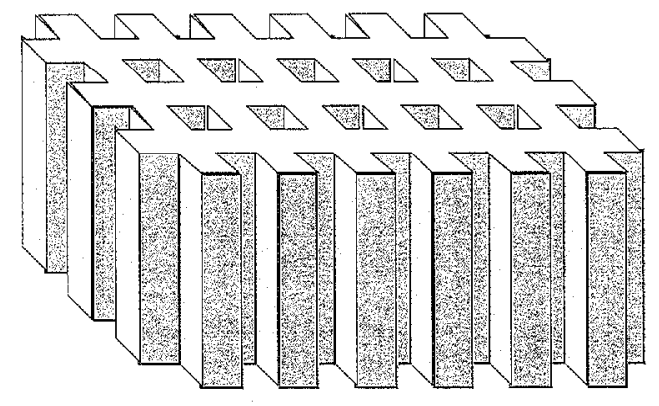

Figure 3. Perspective drawing of the micromachined silicon screen.

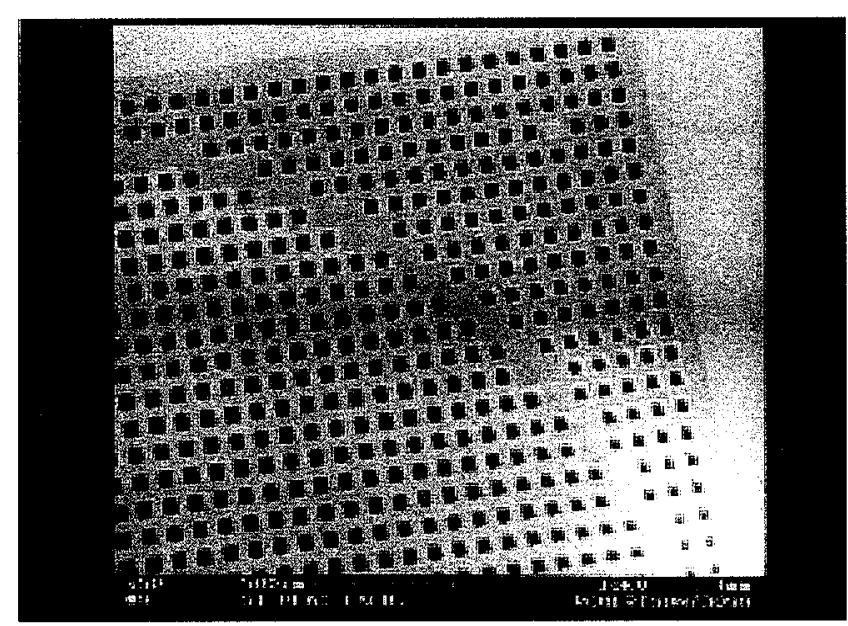

Figure 5. SEM photograph showing a portion of one silicon screen. The circular section is positioned over the zeolite filled separation column and contains the resistive heater. The corners of the structure have also been micromachined to reduce the thermal mass of the surrounding silicon.

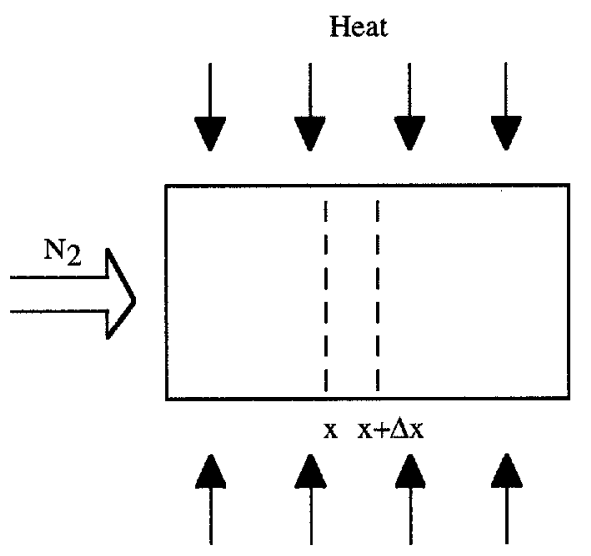

Figure 7. Control volume depicting the heat transfer through one channel.

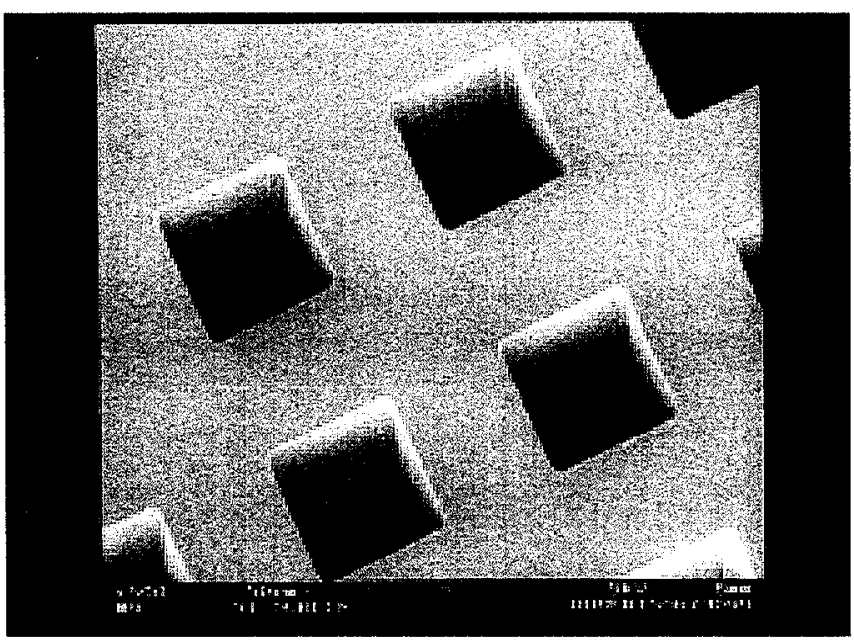

Figure 4. SEM photograph of the DRIE through holes as viewed from the frontside of the wafer. (Plasma entrance holes)

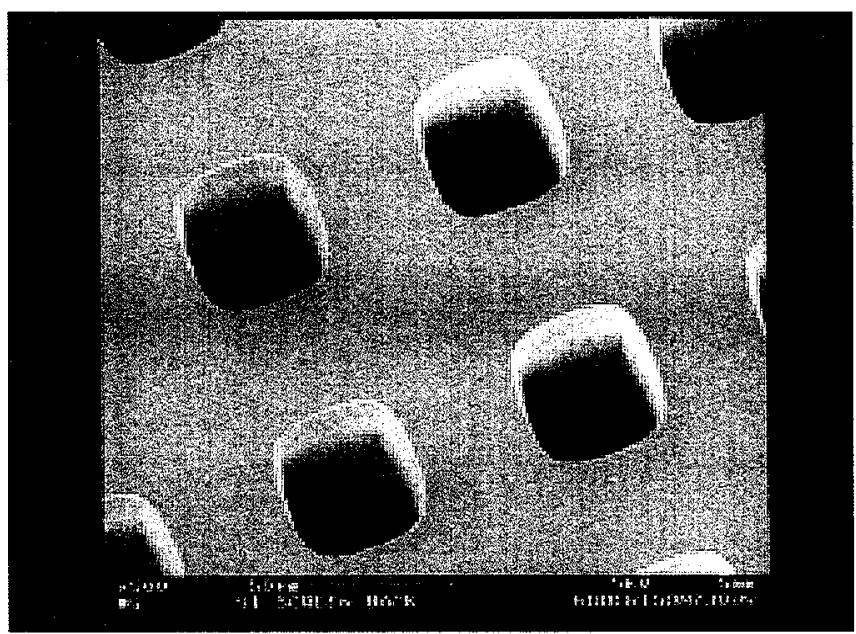

Figure 6 SEM photograph of the DRIE through holes as viewed from the backside of the wafer. (Plasma exit holes)

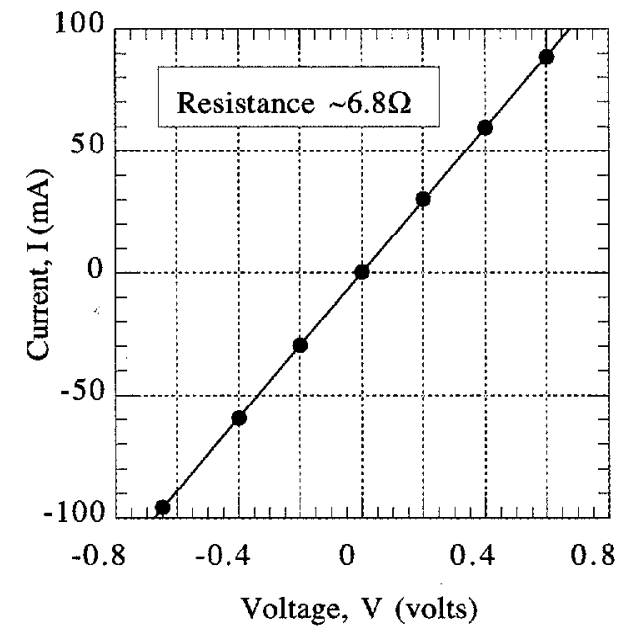

Figure 8. Vertical resistor IV characteristic measured with the substrate floating. 
calculated resistance of the $\mathrm{Al}$ interconnects is $<0.025 \Omega$. A plot of the IV characteristic is shown in Figure 8. The vertical resistor $\mathrm{L} / \mathrm{W}$ ratio is approximately 1.8 . Therefore, the average resistance of each channel is between $47 \mathrm{~K} \Omega$ and $55 \mathrm{~K} \Omega$. The average sheet resistance is between $26 \mathrm{~K} \Omega /$ square and $31 \mathrm{~K} \Omega /$ square. However, sheet resistance measurements on an unetched control wafer yielded $\sim 177 \Omega$ /squarc. It is possible that although significant doping occurred on the surface of the wafer (and on the surface of the control wafer) that the doping density was reduced inside the channels resulting in a larger sheet resistance for the micromachined channels.

With the substrate grounded the total resistance of the heater die was $\sim 5 \Omega$. The decrease is due to a large area $\mathrm{pn}$ junction diode fonned between the $n+$ resistor and the $p$ substrate. The parasitic device should not substantially impact the operation of the heater.

At steady state, approximately $8.24 \mathrm{~mW} / \mathrm{screen}$ (or $32.96 \mathrm{~mW} /$ die) is required to heat $1 \mathrm{SCCM}$ of $\mathrm{N} 2$ gas from $25^{\circ} \mathrm{C}$ to $400^{\circ} \mathrm{C}$. Therefore, assuming an average resistance of $6.5 \Omega$, a voltage of $\sim 0.46 \mathrm{Volts}$ is required at steady state.

\section{CONCLUSIONS}

A vertical micromachined resistive heater for a micro gas separation column has been designed and fabricated. The measured vertical resistance is between $6 \Omega-7 \Omega$. The calculated power required to heat the purge gas at steady state is $\sim 33 \mathrm{~mW} /$ die requiring a applied bias of $\sim 0.46 \mathrm{volts}$ and a current of $\sim 71 \mathrm{~mA}$. The calculated heat transfer coefficient is $\sim 3000 \mathrm{~W} / \mathrm{m}^{2} /{ }^{\circ} \mathrm{C}$.

The micromachined resistive heater presented in this paper is a demonstration of the type of vertical integration that is possible using deep reactive ion etching. The resistor diffusion occurred after the Bosch etch, thus forming the resistor along the micromachined surface. The diode is an insignificant parasitic device in this structure, but again demonstrates the use of the vertical dimension of the silicon wafer to produce a semiconductor device.

\section{ACKNOWLEDGEMENTS}

The author would like to gratefully acknowledge the support provided by the National Science Foundation under contract 9720493. The author would also like to express her thanks to Mr. Ray Filosov and Dr. Floyd Miller for their assistance with the fabrication of these devices at the Sherman Fairchild Center for Solid State Studies at Lehigh University. In addition, the author is grateful to Mr. John Williams for his assistance with the Bosch etching at Cornell University.

\section{REFERENCES}

1. J. K. Robertson, and K. D. Wise, "A Low Pressure Micromachined Flow Modulator", Sensors and Actuators:A. Physical, 71/1-2, pp. 98-106, (1998).

2. E. Meng, X. Q. Wang, H. Mak, and Y. C. Tai, "A CheckValved Silicone Diaphragm Pump", Proc. IEEE The Thirteenth Annual International Conference on Micro Electro Mechanical Systems, Miyazaki, Japan, Jan. (2000), pp. 62-67.
3. N. P. Chopey, G. Ondrey, and G. Parkinson, "Microreactors Find New Niches," Chemical Engineering, pp. 30-33, March (1997).

4. J. D. Parker, J. H. Boogs, and E. F. Blick. Introduction to Fluid Mechanics and Heat Transfer, Addison-Wesley Publishing, Reading, MA (1974), p. 190. 